\title{
Article \\ Heat Engines of Extraordinary Efficiency and the General Principle of their Operation
}

\author{
Remi Cornwall
}

University of London Alumni Society, Malet Street, London WC1E 7HU, UK; r.o.cornwall@qmul.ac.uk

\begin{abstract}
The intention of this paper is to elucidate new types of heat engines with extraordinary efficiency, more specifically to eventually focus on the author's research into a temporary magnetic remanence device. First we extend the definition of heat engines through a diagrammatic classification scheme and note a paradoxical non-coincidence between the Carnot, Kelvin-Planck and other forms of the 2nd Law, between sectors of the diagram. It is then seen, between the diagram sectors, how super-efficient heat engines are able to reduce the degrees of freedom resulting from change in chemical potential, over mere generation of heat; until in the right sector of the diagram, the conventional wisdom for the need of two reservoirs is refuted. A brief survey of the Maxwell Demon problem finds no problem with information theoretic constructs. Our ongoing experimental enquiry into a temporary magnetic remanence cycle using standard kinetic theory, thermodynamics and electrodynamics is presented - yet a contradiction results with the 2nd law placing it in the right sector of the classification diagram.
\end{abstract}

Keywords: Maxwell Demon; phase transition; Ferrofluid; Magneto-calorific effect; time's arrow

\section{Introduction}

We wish to show that the heat engines can run from only one reservoir against prevailing wisdom. However, the argument regarding heat engines would seem pretty closed: in the ideal (with extremal trajectories in T-S space) the working substance is taken around a cycle, placing it in contact with an upper and lower reservoir - adiabatics are alternated with isothermals. This leads to the inevitable conclusion that heat must be rejected to the lower reservoir. If the sequence wasn't followed, we wouldn't return to the initial co-ordinates.

The lynch pin of the two reservoir Carnot cycle is that the working substance has an exact differential relation around the cycle. We shall see later in this paper that, if the working substance after an isothermal and then an adiabatic step, could execute a trajectory different than the repertoire of trajectories provided by an exact differential relation, then it can extract heat energy from one reservoir. The way, as we shall see in section 2 , is to make the working fluid appear temporarily to be a different material - to make it undergo a phase transition, the trajectory will be inexact in part of the cyclical operation.

It is not often thought that batteries, fuel cells, biological life (e.g. a muscle) can be considered as heat engines. The popular view is that heat engines must have some "furnace" (some combustion process) as the hot reservoir and then the cold reservoir to complete cyclical operation. This definition is semantically limited; one must focus on the word "heat", if one is more liberal but then at the same time more precise with the definition:

Heat engines transform one form of energy to another with a substantial change in the entropy of the process that is intrinsic to its operation.

We find it will encompass conventional cyclical engines, batteries, fuel cells, even cellular life too. The term "intrinsic" needs clarification: A charged capacitor discharging into an electric motor is an engine but not an heat engine, though inevitably there will be friction and the generation of heat. Theoretically the loss processes could be minimised - electrical, magnetic hysteresis, bearing or even air friction but a true heat engine would have some change between the standard entropies of 
reactants and products, that can't be minimised by design. This would include rejection or absorption of heat from the environment in performing its task of energy conversion, including that of interconversion of heat too.

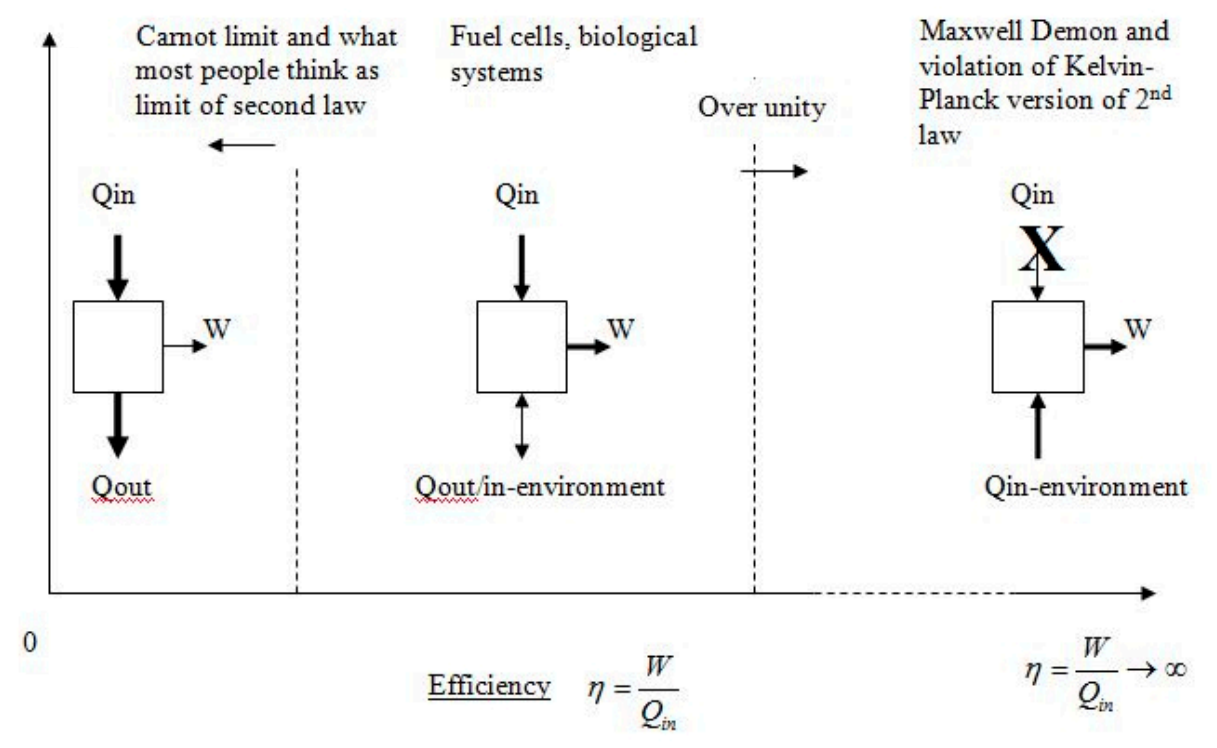

Figure 1. The continuum of heat engines.

Figure 1 makes the assertion that amongst heat engines, that there is a continuum from pure heat conduction, to Carnot limit engines, to fuel cells and biological systems to Maxwell Demon processes[1, 2]. The chart shows from the point of view of efficiency, how particular types of engine fit into the continuum scheme. Logically to the left at zero efficiency, is where heat conduction is placed.

Next come the Carnot cycle limited engines, where some useful work is delivered up to their efficiency limit. In the middle sector of figure 1, we insist (for the argument given previously about the change in entropy) must be the position of batteries, fuel cells and biological systems as heat engines. We have the conundrum that a battery or muscle can still function when the environment (the supposed cold sink) is at an higher temperature than the "engine". It is known that biological systems exceed Carnot efficiency and indeed, E. T. Jaynes[3] in a contentious unpublished work, took the Carnot reasoning applied to a muscle to an illogical conclusion: that living muscles must be operating at some $6000 \mathrm{~K}$ to achieve their work output. Correctly Jaynes points out that the degrees of freedom for the release of chemical energy are very curtailed, unlike the random motion of a fluid in a Carnot cycle being cohered by a piston to linear motion, muscles fibres extend and contract in one very specific direction under the control of ATP. Try as one might to deny that fuel cells and biological systems aren't heat engines, one cannot deny the change in entropy of the reactants.

The diagram (fig. 1) makes clear that one can utilise heat energy much more subtly than a Carnot cycle. The continuum from the middle ground and especially biological systems to Maxwell Demon type processes becomes apparent. Moving to the limit of the middle sector of figure 1, Mae-WangHo[4] has argued that some biochemical processes (especially membrane processes) utilise random thermal motion to achieve more than can be explained by conventional thermodynamics. Thus in the right sector of figure 1, we include the possibility where there is no input heat and the work is achieved wholly by the conversion of environmental heat energy input - a Maxwell Demon. Furthermore we can see that several definitions of the $2^{\text {nd }}$ law [5] do not coincide by this diagram:

- Carnot theorem: All Carnot engines operating between the same two temperatures have the same efficiency and this is always less that unity. 
- Kelvin-Planck: No device, operating in a cycle, can produce the sole effect of extraction a quantity of heat from a heat reservoir and the performance of an equal quantity of work.

- Clausius: No process is possible for which the sole effect is that heat flows from a reservoir at a given temperature to a reservoir at higher temperature.

These considerations of non-coincidence in the definitions, alone, should make us question or find a flaw in the Carnot theorem. The next section shall do just that.

\section{Discussion: The general principle of heat engines of extraordinary efficiency}

Here we shall show that heat engines constructed from materials undergoing a phase transition, permit the development of extraordinarily efficient heat engines. To begin, let us consider microscopic heat flows. Microscopic fluxes at equilibrium have an exceedingly large amount of microscopic work occurring at constant temperature, as this clearly is how individual particles can rise and fall in a potential field at equilibrium. There is no conflict with the Carnot result if one takes this viewpoint, that as the temperature difference between the reservoirs tends to zero, the efficiency tends to zero: we argue that the microscopic work-flows at constant temperature become essentially limitless, based on the microscopic heat-flows, which are essentially limitless too, $\eta=\left(Q^{+}-Q^{-}\right) /\left(W^{+}\right.$$\left.\mathrm{W}^{-}\right) \rightarrow 0$, where the positive superscripts represent large, finite heat or work into the system and the negative superscripts represent large, finite flows out. This is guaranteed by the statistical fluctuation of temperature at equilibrium[6, 7], as depicted in figure 2.

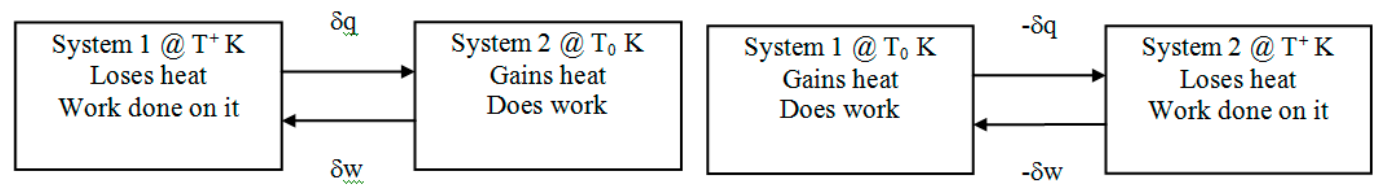

Figure 2.Statistical fluctuation in temperature with micro-heat and micro-work flows.

It becomes apparent why phase change is the key to making a Maxwell Demon. At the phase boundary at equilibrium, microscopic fluctuations in temperature effectively form microscopic heat engines that are able to do work against the phase boundary.

Lemma: Constant temperature microscopic work theorem

At constant temperature microscopic heat and work are available and can partition energy across a phase boundary.

So if a microscopic demon is possible how is a macroscopic demon made?

Lemma: Phase Transition Sorting

Macroscopic work is potentially obtainable from microscopic work processes at constant temperature by the working substance undergoing a phase transition.

By definition, a phase is a macroscopic representation of underlying microscopic properties. In a sense, the phase change has "magnified" the microscopic demon. We say "sorting", as the energetic molecules have partitioned across the phase boundary, as distinct from the less energetic. This can be understood from the thermodynamic identity:

$$
d U=T d S-P d V+\mu(P, T, \phi) d n
$$

where $\mu$ is the chemical potential, $\mathrm{n}$ the number of particles, $\phi$ is a potential function (of position, particle number etc.)

As mentioned in the introduction, $\mathrm{dU}$ is an exact integral; any means of cycling the working substance by any of the variables of the system, will not produce excess energy from the lowering of the internal energy of the working substance. Let us understand this more by reviewing a conventional Carnot engine: The working substance being only one material is constrained to traverse fixed trajectories in P-V or T-S space (fig. 3). The familiar alternating of isothermals with adiabatics is required to map out an area, as moving reversibly along 1-2: isothermal-adiabatic or 1- 
2-3: isothermal-adiabatic-isothermal, will not return to the starting co-ordinates. The last process, 4 , maps out an area so that changes in heat are equal to changes in work: $d U=0 \Rightarrow \Delta Q=\Delta W$. This cannot be done with just one reservoir and the last step 3-4 must come into contact with the lower reservoir.
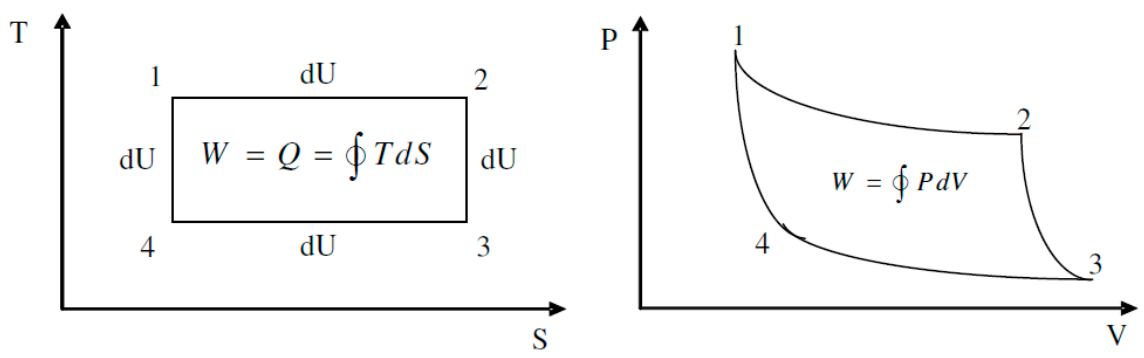

Figure 3. P-V and T-S diagram of Carnot heat engine or refrigerator

Is there a way around this two reservoir argument? Consider now the meaning of the chemical potential, it is the thermodynamic potential per particle:

$$
d u=T d s-P d v+\mu d n
$$

The lower case indicates that this is per particle. The chemical potential has two parts, the internal and external[8]; the sum is often called the total chemical potential. If at some point in a thermodynamic cycle an external potential $\mu_{\text {ext }}$ is added or changed then the thermodynamic identity can be made inexact, relative to the old expression for the identity,

$$
\delta u^{\prime}=T d s-P d v+\mu_{\text {int }} d n+\mu_{e x t} d n
$$

The condition for exactness for du would require $\mu=\left(\frac{\partial u}{\partial n}\right)_{s, v}$. Now, if $\mu_{\text {total }}=\mu_{\text {int }}+\mu_{\text {ext }}$ then $\mu_{\text {total }}=\left(\frac{\partial u^{\prime}}{\partial n}\right)_{s, v} \neq\left(\frac{\partial u}{\partial n}\right)_{s, v}$ Thus if any external potential is added and results at some point in the cycle in a change of the total chemical potential, the resulting thermodynamic potential du' will be rendered inexact compared to $\mathrm{du}$. It is as though we have a different working substance not constrained to the trajectories of one substance in P-V or T-S space (fig. 4), that at some part of the cycle reverts to the original substance (without doing the trajectory 2-3 in reverse) to complete the cycle, so we can achieve net work from only one reservoir. Considering the ideal heat engine argument and the extremal trajectories (from the point of being adiabatic and only doing work or isothermal and only transferring heat), at step 2-3 there is a process performing work and absorbing heat that isn't polytropic (and so in contact with a reservoir).

A change of $\mu$ can only correspond to a phase change as this will introduce potential energy terms, such as that pertaining to latent heat (discontinuous $1^{\text {st }}$ order, see appendix) or new magnetisation energy terms (continuous $2^{\text {nd }}$ order), for instance, i.e. dipole-work (section 4).
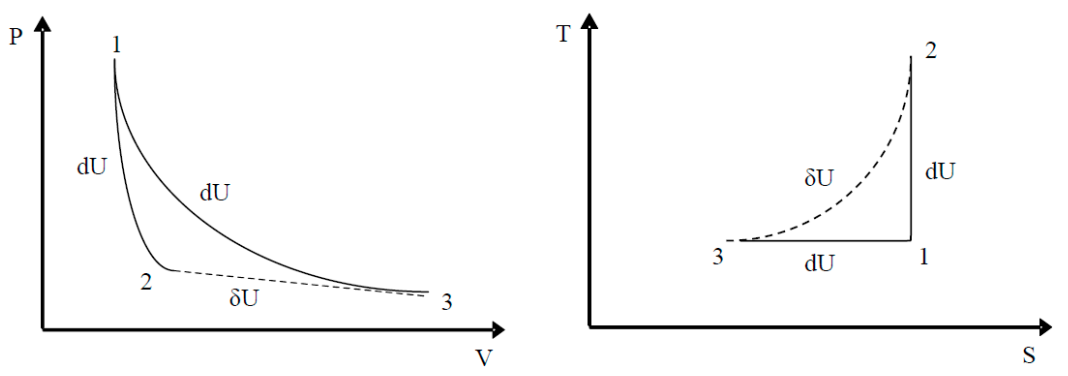

Figure 4. Illustrative P-V and T-S diagrams for an one reservoir engine.

Appendix 1 contains a probably impractical setup of $1^{\text {st }}$ order phase transition Maxwell Demon (figure 5a) thought of by the author in his early endeavours. Later (section 4 ) we shall present a $2^{\text {nd }}$ order phase transition demon which will possibly lead to practical power generation on a large scale. 
This demon is a "reverse demon": rather than the randomness of heat being sorted directly (as by a $1^{\text {st }}$ order phase transition demon), a volume of working substance is ordered and then randomised. The step of randomisation is made to couple to some other system, such that the randomisation is forced to perform macroscopic work; figure $5 b$ illustrates this concept. Other bold claims of extraordinarily efficient heat engines are beginning to be cited in the literature base [5, 9-12] and if these are cyclical thermodynamic processes (as opposed to kinetic - say in rarefied gases), then they must be operating by a change in chemical potential to render the thermodynamic potential inexact around a cycle.

Having challenged the Carnot formulation of the $2^{\text {nd }}$ law, we should now seriously look at the status of it and just how demons could work, given the literature against it.

$\underline{1^{\text {st }} \text { order: (See appendix } 1 \text { and "phase changing catalyst") }}$

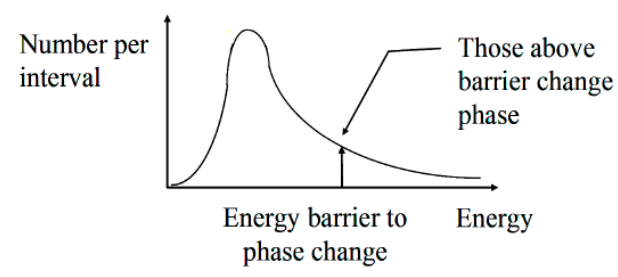

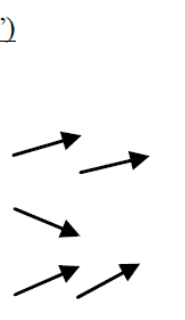

1) Magnetic field orders part of the system.

Energy Input $=\mu_{0} H M V$

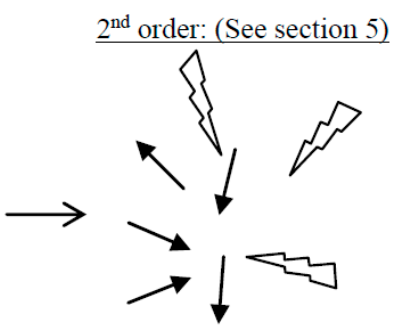

2) Buffeting from rest of system.

Energy out $=\frac{1}{2} \mu_{0} M^{2} V$

Figure 5 a,b. $1^{\text {st }}$ order and $2^{\text {nd }}$ order phase transition "reversed sorting" demon

\section{Discussion: Real Maxwell Demons from phase transitions}

The introduction of Clausius' integrating factor $(1 / \mathrm{T})$ into the thermodynamic identity lead to the discovery of an exact property of systems, the entropy[1, 8, 13]. This was soon realised by Boltzmann to be related to the microstates of the system[1,8]. Contemporaneous developments in statistical mechanics and kinetic theory elucidated notions of molecular chaos and the approach to equilibrium, in the thermodynamic limit, by Boltzmann's H-Theorem[7]. It would seem perverse to question the $2^{\text {nd }}$ law for the principle of ergodicity; in the long run, things randomise, how can they un-randomise? A sorting process is needed and this invokes notions of the celebrated Maxwell Demon[1, 14].

In the intervening years of debate started by Maxwell, the counter-arguments condensed into three areas:

- $\quad$ The Demon would be blind in black-body radiation to see anything. Kirchhoff[15];

- A mechanical sorting element of size commensurate to the molecules to effect the sorting gets randomised too, until ineffective. Smoluchowski[14], Feynman[16] 'Ratchet and Pawl';

- $\quad$ Or to perform sorting, it would require measurement and expenditure of energy for storage of information. Szilard[2], Brillouin[15], Landauer/Bennett[17].

The last consideration defined Information Theory, as the first half of the $20^{\text {th }}$ century saw the development of the digital computer - this Demon wouldn't have to be supernatural but a finite state machine, with the concomitant use of energy and dissipation to heat. Ironically the very thing it was trying to do, lower entropy, would cause more production of it.

It can be seen that the problem with the Demon is the need to construct something that measures, keeps the processing state of the machinery and then acts effectively. It is pointless to try and build anything mechanical to affect sorting; however by the analysis of the previous section, the energy barrier due to material phase acts at a microscopic level between molecules, it intrinsically measures and "keeps the state of the machinery" and then partitions/sorts molecules by kinetic energy.

The theory developed shall now be applied, with a description of the author's research into a $2^{\text {nd }}$ order phase change demon, made with a magnetic fluid as the working substance. 


\section{Discussion and Results: A temporary magnetic remanence cycle with extraordinary efficiency projections}

It has been discussed previously in this paper how heat energy will partition across a phase boundary. The Zeroth law would have two adjacent phases at thermodynamic equilibrium, yet the chemical potential would show one phase as having an higher potential than the other. One might consider a phase as an "halfway demon" (fig. 5a), having "harvested" the heat energy (microscopic randomised energy), what remains is to liberate macroscopic work from the higher energy phase. In the case of a discontinuous $1^{\text {st }}$ order transition, the latent heat, in the form of a temperature rise of the working substance and potential energy from the change in density was considered (appendix 1). In the latter consideration regarding potential energy, it can be argued that the external chemical potential (eqn. 3) makes the material form a continuously changing phase in the rising vapour phase part of figure 10 (appendix 1), so this is a gravity sorting demon.

The continuous order-disorder the transitions of $2^{\text {nd }}$ order phase changes afford another means of making a sorting demon. It was decided to pursue a more practical $2^{\text {nd }}$ order device that would allow the direct conversion of heat energy to electricity[18]. The starting point for analysis is the thermodynamic identity of a magnetic system:

$$
d U=T d S+P d V+\mu_{0} H d \mathrm{M}
$$

The magnetic work term is composed of the intensive magnetising field variable, $\mathrm{H}$ and the extensive magnetisation variable, $M$. The research studied a magnetisation-demagnetisation cycle of a super-paramagnetic material[18, 19] called ferrofluid, which had the aim of (slowly) magnetising the ferrofluid and then abruptly removing the magnetising field to leave a temporary remnant flux. The last aspect of this cycle distinguishes it from other magnetic heat engines: usually the field is continuously applied at one value, then another, whilst the working substance is placed in contact with the upper and then lower reservoirs; variation in magnetic entropy absorbs or rejects heat energy[19]. The magnetic work is cyclical, such that the absorbed heat is usually then expressed as pressure volume work, for instance, the magneto-calorific ferrofluid pump[19] (or figure 10 ref. [18]). In our temporary remanence, magnetisation-demagnetisation cycle, we aren't concerned with pressure-volume work and use the following identity:

$$
d U=T d S+\mu_{0} H d \mathrm{M}+\mu_{0} K_{e} M d \mathrm{M}
$$

where a new work term, called the dipole-work (appedix 4, ref. [18]), reflects the work done by the temporary remnant flux into an electrical load, on removal of the magnetising field by the working substance. The figures 6i-iii illustrate the basic concept and a plant diagram.
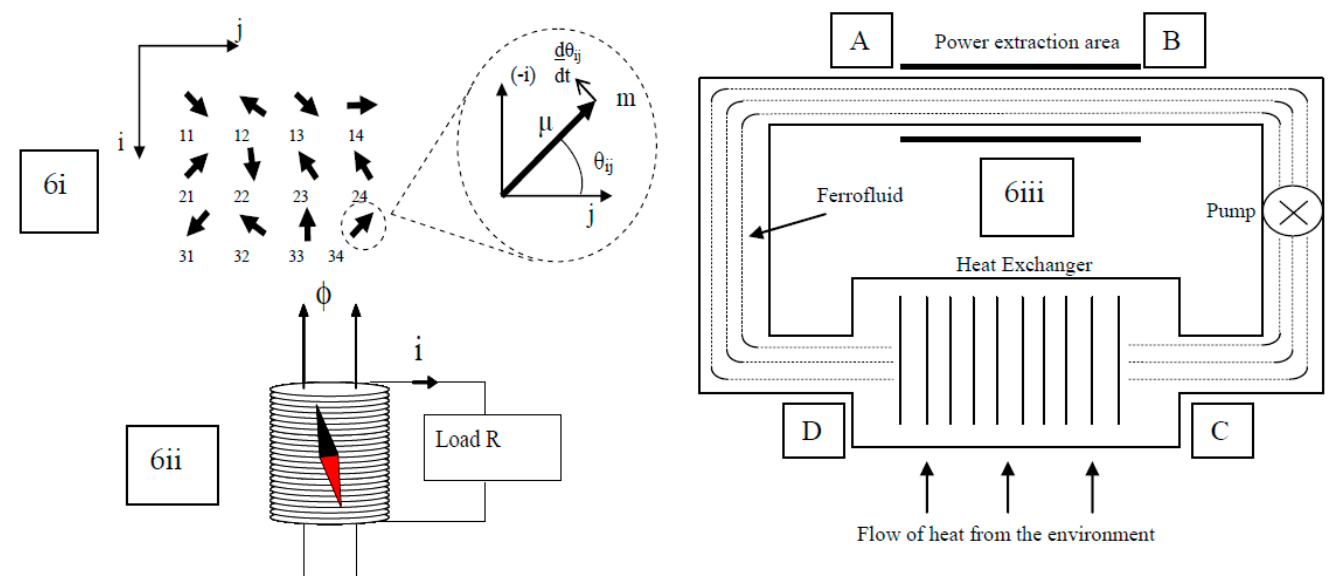

Figure 6i,ii,iii. The basic flux collapse power generation method and plant diagram.

Figure 6i shows an ensemble of magnetic dipoles, representing the ferrofluid working substance in a matrix for kinetic computer simulation and this will be discussed shortly. 
Figure 6ii conceptually shows how the decaying flux, from the multitude of nanoscopic ferrofluid working substance dipoles, looks just like a rotating bar magnet (section 2.1.1, ref. [18]), whereupon it directly generates an electric current into an electrical load.

Figure 6iii shows the plant diagram for the process: in the power extraction area A-B, many magnetisation-demagnetisation cycles occur per second, such that the collapsing flux in the region A-B delivers electrical work by induction into a coil around the region. It is of note too that if the magnetisation is bipolar, no net magnetising moment is expressed in the region, such that pressurevolume work becomes negligible. The working substance cools in the region A-B as it performs electrical work into the load and so it is pumped around to the heat exchanger in region C-D, to go around the circuit once again.

The thermodynamic analysis is closely based upon the magneto-calorific effect (section 2.2 and appendices 6, 7 ref. [18]) in close proximity to a reservoir (in this case the suspending fluid of the magnetic particles in the ferrofluid). The magnetic work term $\mu_{0} H d \mathrm{M}$ (eqn. 5) also reflects the chemical potential of the working substance, as the magnetisation $\mathrm{M}$, indicates the quantity of species in a phase, as does the dipole work $\mu_{0} K_{e} M d \mathrm{M}$ term too, which is an external work term (eqn. 3). In a nutshell for a symmetric case with no dipole work, like eqn. 4 , we find that upon applying a magnetic field, heat is rejected from the magnetic particles in the ferrofluid to the suspending liquid and upon turning the field off, the same heat is returned from the fluid.

However for the asymmetric case on the demagnetisation step, if the magnetic system is coupled by induction to the output coil (fig 6ii) and if the magnetising field is switched off abruptly (appendix 8 ref. [18]), not only is the magnetising energy returned but additional dipole-work results from a lowering in temperature and entropy of the working substance by the conversion of heat energy (section 2.2.2 ref. [18]).

\subsection{Kinetic theory model}

The conversion of heat energy comes from the thermal buffeting of Brownian motion (fig. 5b). A kinetic theory model can both analytically and numerically prove the lowering of temperature. Regarding figure $6 \mathrm{i}$, a model of dipole-dipole interactions leads to the angular acceleration of each dipole, with I the moment of inertial, $\mathrm{k}_{\mathrm{dip}}$ a coupling constant, $\mathrm{m}_{\mathrm{ij}}$ the dipole moment at array position $\mathrm{ij}$ :

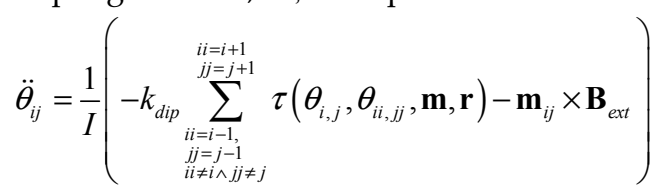

The torque experienced by each dipole is from the external field of the solenoid (Bext) and the dipole-dipole interactions resulting from the local fields of its neighbours:

$$
\tau\left(\theta_{i j}, \theta_{i i, j j}, \mathbf{m}, \mathbf{r}\right)=-\mathbf{m}_{i j} \times \overline{\mathbf{B}_{\text {local.neighbour }}}
$$

This equation (7) taken as a bulk effect over the whole lattice, is of the form const $\mathrm{x}$ MdM or the dipole-work, where $B_{\text {local.neighbour }}=\mu_{0} M$. The model can be run as a molecular dynamics simulation (the

Matlab code is in appendix 10, ref. [18]) and the author attempted this to good success, apart from the lack of convergence or energy drift in these type of simulations from use of non-sympletic algorithms[20]. It wasn't thought worthwhile to pursue this further when, as we shall see, analytical solution exists. Furthermore, energy drift adds energy to the system and yet the simulations show temperature and entropy reducing, despite this. The entropies of position and velocity and the temperature can be calculated from the simulation data:

$$
\begin{aligned}
& S_{p o s}=\text { const } \times \ln \left(\text { standard deviation } \theta_{i j}\right) \quad(\text { positional) } \\
& S_{v e l}=\text { const } \times \ln \left(\operatorname{standard} \text { deviation } \dot{\theta}_{i j}\right) \quad \text { (velocity) } \\
& T=\text { const } \times \text { average }\left(\dot{\theta}_{i j}^{2}\right) \quad(\text { from kinetic energy) }
\end{aligned}
$$




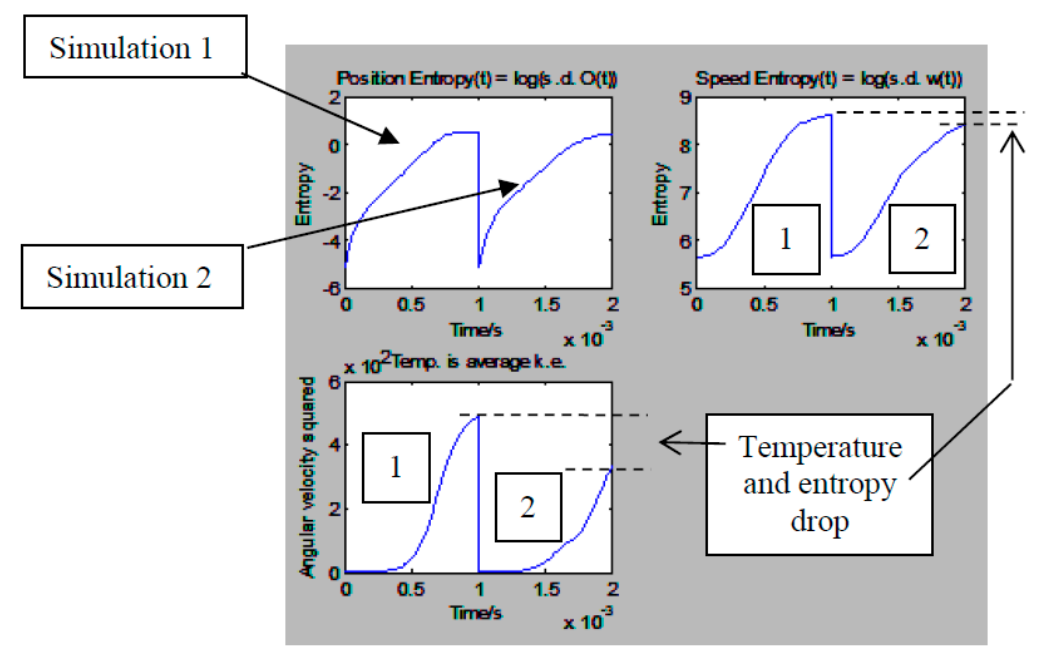

Figure 7. Two back-to-back simulations: Relaxing to equilibrium and then relaxation to equilibrium with dipole work.

Two simulations were performed (labelled 1 and 2 on diagram), one after the other: In the first simulation the dipoles were all aligned at the start with zero kinetic energy. The simulation shows this "relaxing" to a random orientation (the position entropy increases). The potential energy at the start is converted into random kinetic energy (hence the temperature rises, as does the velocity entropy). It is interesting to note that the model randomises itself without any random input. This is just the chaotic dynamics of the three (or more) body problem - the reason why ordered energy degrades to heat energy.

The second simulation follows right after the $1^{\text {st }}$ for comparison, models relaxation with dipolework, that is, the assembly generates electrical work which leaves the system and gets dumped into the resistive load. An analytical solution can be obtained (section 2.1.2 ref. [18]) by the statistical averaging of the ensemble eqn. 6 :

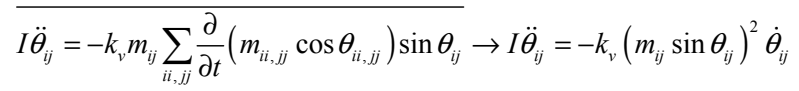

Thus each dipole experiences a drag force (hence proportional to the angular velocity $\dot{\theta}_{i j}$ ) and slows (hence both temperature and velocity entropy decrease) and this is directly related to the dipole-work (eqn. 5). This shows the mechanism for the transduction of heat energy from the working substance to the electrical load.

\subsection{Sundry considerations}

There are a few subtle but non-fundamental electrodynamic considerations (Cornwall [21], section 4, appendices 8 and 9, ref. [18] ), which would properly be called technical electrical engineering type matters, related to cancelling the re-magnetising field from the induced current. The so-called "H-field cancellation circuit" is required to ensure that the dipole-work can exceed the magnetising energy input.

Power losses are also modelled with the most notable being the working substance's (ferrofluid) dissipation on applying a field (appendix 5, ref. [18] ). The physical aspect of ferrofluid relaxation and heating effects is closely related to the fluctuation-dissipation theorem[6, 7] but it is sufficient to say that, if the magnetising field is turned on more slowly than the relaxation rate of the ferrofluid, these losses become vanishingly small. Other losses such as pumping losses can be minimised by design and it was already mentioned that, if the magnetising field is bipolar, there will be no net ferrofluid pump effect[19] into the region A-B (fig. 6iii), for which work would have to be expended. Hysteresis or "anomalous" magnetic losses are quantifiable and minimal, as too are electrical I'R losses by design. 
9 of 16

\subsection{Plant T-S diagram and expected power levels}

The device consists of two conceptual cycles: the multitude of magnetisation-demagnetisation cycles on the working substance that occur in region A-B of figure 6iii, called the "micro-cycle" and the overall flow of the working substance in the circuit A-B-C-D, called the "macro-cycle" (sections 2.2.2 - 2.2.4 ref. [18]). The micro-cycle T-S diagrams are complicated but it is essentially enough to say that in region $\mathrm{A}-\mathrm{B}$, temperature and entropy decrease, as shown in the kinetic simulation too.

In the region $A-B$, all the temperature and entropy lowering of the micro-cycles act to traverse path A-B in figure 8. Compared to the path C-D, which represents the working substance heating up as it passes through the heat exchanger (fig. 6iii), the path A-B behaves as though the working substance has an higher, or "virtual" heat capacity, $\mathrm{C}_{\text {virt }}>\mathrm{C}_{\mathrm{H}}$; it looks like a different working substance. The reasoning is this: if instead the path B-A was traversed on the T-S diagram, warming it up with be more difficult and this is not surprising, the substance has been coupled to an external system to which it loses energy. Around the cycle to A-D, we come back to the initial thermodynamic co-ordinates and thus the changes in entropy can be related to the work extracted.

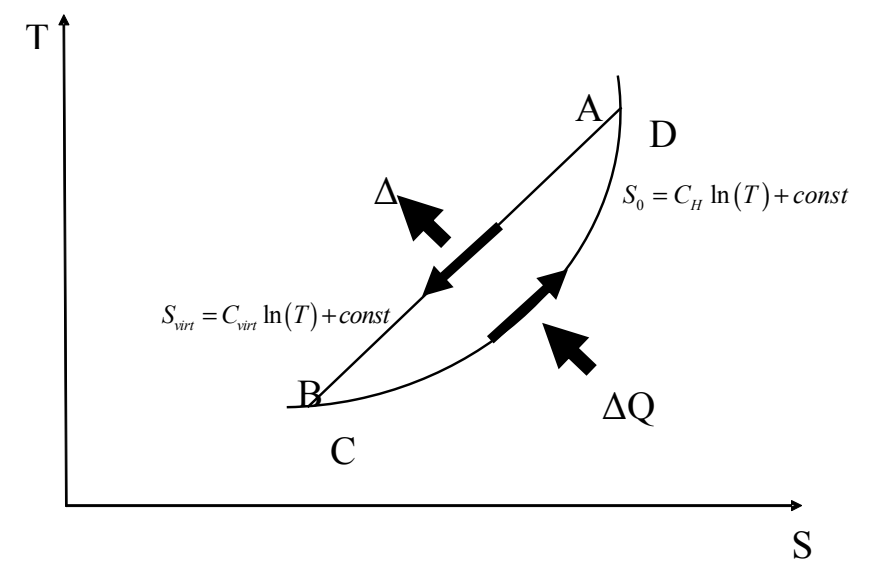

Figure 8. Macro-cycle T-S diagram related to points on plant diagram.

Expected power levels are large, in the region of mega-Watts per $\mathrm{m}^{3} / \mathrm{s}$ flow rate of the working substance (section 2.2.3. ref. [18]). This results from a Fourier heat conduction law calculation for, the amount of heat per unit volume by the magnetic ferrofluid particle suspended in the said volume:

$$
\begin{aligned}
& \frac{\partial q}{\partial t}=-k_{\text {fluid }} \frac{\partial T}{\partial x} \\
& \text { where } Q=\iint_{A, t} q d A d t
\end{aligned}
$$

The heat conductivity of the suspending fluid is kfluid and $\mathrm{dA}$ is an infinitesimal surface area normal to the heart flux q. The time scale is of the order of the relaxation, $\tau$, of the magnetic flux of the ferrofluid and so we write, with Asurf the combined surface area of all the ferrofluid particles:

$$
\Delta w \text { per volume }=\Delta Q=-k_{\text {fluid }} \tau \frac{A_{\text {surf }}}{\Delta x} \Delta T
$$

The temperature change $\Delta T=\Delta T_{o n}-\Delta T_{\text {off }}$ comes from the magneto-calorific effect (appendix 1 , ref. [18]), where $\Delta T_{\text {on }}$ represents the temperature excursion on switching on the field (positive) and $\Delta T_{\text {off }}$ when the field is switched off (negative),

$$
\Delta T_{\text {on }}=-\mu_{0} \frac{T}{C_{H}}\left(\frac{\partial \mathcal{M}}{\partial T}\right)_{H} \Delta H
$$

where: $\mu_{0}$ is the permeability of free space, $\mathrm{T}$ is the absolute temperature, $\mathrm{C}_{\mathrm{H}}$ is the heat capacity, $\boldsymbol{M}=M V$ is the volume magnetisation ( $\mathrm{M}$ is the magnetisation) and $\mathrm{H}$ is the magnetic field strength. 
10 of 16

The magnitude of the temperature change on switch off would be the same as switch on but for the dipole work term $K_{e} \Delta M$ (eqn. 13a, where $\mathrm{K}_{\mathrm{e}}$ is the electrical coupling of the change in the dipole flux to the external electrical load),

$$
\begin{gathered}
\Delta T_{\text {off }}=-\mu_{0} \frac{T}{C_{H}}\left(\frac{\partial \mathcal{M}}{\partial T}\right)_{H}\left(\Delta H+K_{e} \Delta M\right) \\
\Rightarrow \Delta T_{\text {off }}=-\mu_{0} \frac{T}{C_{\text {Virtual }}}\left(\frac{\partial \mathcal{M}}{\partial T}\right)_{H} \Delta H
\end{gathered}
$$

In eqn. $13 \mathrm{~b}$, the dipole work term is absorbed into a similar expression to eqn. 12 by having a "virtual heat capacity" - the work re-partitions the heat energy of the system into the electrical load and makes the magnetic system seem to have a lower heat capacity (if the path A-B is traversed in figure 8 , though on a T-S diagram, logically it appears higher B-A).

The analytical expression below, derived from the Langevin equation (appendix 1, ref. [18]), models $\left(\frac{\partial \boldsymbol{M}}{\partial T}\right)_{H}$ and the change of the magnetic moment of the ferrofluid,

$$
\left(\frac{\partial \mathcal{M}}{\partial T}\right)_{H} \approx-M_{S}(0) \frac{m_{\text {nano }} H}{3 k T^{2}}
$$

where: $M_{S}(0)$ is the saturation magnetisation of the ferrofluid (order of $25,000 \mathrm{~A} / \mathrm{m}$ ) and the magnetic moment of a ferrofluid particle is of the order $1 \times 10^{-25} \mathrm{~J} / \mathrm{A} / \mathrm{m}, \mathrm{k}$ is Boltzmann's constant. $\Delta H$ is of the order $8000 \mathrm{~A} / \mathrm{m}$.

Upon plugging eqn. 14 into eqns. 12 and $13 \mathrm{~b}$ to obtain $\Delta T=\Delta T_{\text {on }}-\Delta T_{\text {off }}$ and then into eqn. 11 with the following realistic values (section 2.2.3. ref. [18]): the order of ferrofluid nano-particles per $\mathrm{m}^{3}$ of suspending fluid is some $1 \times 10^{23}$ particles (Rosenweig [19]), which gives a total surface area of some $3 \times 10^{7} \mathrm{~m}^{2}$ (Asur) for a particle (with surfactant sheaf) of order $25 \mathrm{~nm}$ radius $(\Delta x)$ and then the figures for the heat capacity and conductivity of water, we obtain heat to work conversion figures of the order of $\mathrm{MW} / \mathrm{m}^{3}$ for a change in heat capacity, CVirtual, of about $10 \%$. The thesis text gives justification for this.

\section{Conclusion and discussion: The $2^{\text {nd }}$ Law is universally valid but what is Time's Arrow when $\Delta S=0 ?$}

The universe is a big place and the $2^{\text {nd }}$ law is ultimately global and true. Despite claims that it is not valid[5], this needs to be qualified with the notion that for relatively small regions (compared to the universe) and for relatively small time timescales, it can go into reverse by Maxwell Demon processes. A demon device in a closed, isolated environment (section 4 and fig. 10, appendix 1) presents a paradox: processes that generate entropy are balanced by those which reduce it. Presumably the demon device could generate enough excess power to operate a maintenance automaton, thus allowing this state of affairs to persist indefinitely. The only constraint would be whether the automaton could have enough time to refine and reconstitute materials in the device, as they denude from it into the environment (reversing the $2^{\text {nd }}$ law tendency for erosion and dispersal) and that the computer code operating the automaton doesn't become corrupted by thermal motion. The total entropy change for the macroscopic system comprising the device, repair automaton and the closed isolated system could be kept at zero, indicating no time evolution of the system - much as the microscopic equilibrium state, so whither the $2^{\text {nd }}$ Law and Time's Arrow?

Should we be so scared by the concept of type 2 perpetual motion? The demon device in the setting discussed is not really perpetual but probabilistic - its code could fail by Boltzmann thermal disruption. Furthermore, as it repairs itself, the modifications will not be precisely the same as to what went before but wholly acceptable and functional to the specification of the device: If the control unit of the demon device had a transistor to be replaced by the repair automaton, it matters not that an impurity dopant in a p-n region of the transistor is in one crystal plane than another, rather, that the bulk properties are consistent with the functioning of the device (not to mention simply printing the serial number of the device in a different font, to be flippant). To be "perpetual" must mean to be perennially the same, by definition. To the point, looking at cosmological time-scales, how can 
thermodynamic abstractions on the states of matter be generally true? There is no "perpetual" in physics, the term should be banished.

In search of a law to describe the time evolution of the condition $\Delta S=0$, we already know that heat energy is microscopic "perpetual" motion with the continual exchange of kinetic to potential energy. Two-body simple harmonic oscillation does this and generalised three-body and n-body motion introduces a chaotic element[7, 22, 23]; we might extend the notion and call it "n-body complicated oscillation". Clearly a demon is part of the n-body complicated oscillatory dynamics of the system and we should find the law to describe macroscopic time evolution when $\Delta S=0$. Such a law already exists and covers all cases when $\Delta S=0, \Delta S>0$ or even if $\Delta S<0$. Moving beyond two bodies, one can characterise the evolution of the separation of two initially close trajectories in phase space by the Lyapunov Exponent[22, 23], $\lambda$ :

$$
\left.|\delta s(p, x)|_{t}\left|\approx e^{\lambda t}\right| \delta s(p, x)\right|_{t=0} \mid \quad \text { eqn. } 15
$$

As such, it represents the loss in information from the state vector. Given the precision we can specify the state vector, once the system evolves the error bounds to which we can specify a trajectory will soon take up a volume as great as the phase space. Even for a god, in a clockwork universe too, such a calculation would be impossible to perform with infinite precision. Although the universe can be described by mathematics, it is not merely a machine; a similar viewpoint was raised in section 3 about real demons, as compared to the information theory construct of one. So at equilibrium with $\Delta S=0$, if it were possible to know a system state vector at some instant to some precision, its predictability will soon be gone. Loss of information is truly the arrow of time.

This leads onto the interesting disparity between microscopic, time reversible processes and macroscopic irreversibility. We believe that there is a category error in relating the real world to a computer simulation: The results of a computer simulation maybe run back and forth as a movie, supposedly reflecting the microscopic reversibility of laws but the computer has given the luxury and false misconception that the state vector is stored, as if on a reel of film. The truth is, physical laws predict differential sequential processes: we move from a time $t_{1}$ to $t_{2,}$, specifically $+t+d t$ or $-t$ $\mathrm{dt}$. It matters not if we use a positive or negative direction of time in the microscopic laws, as long as we use it consistently. The evolution of the state vector will always suffer loss of information, run forward or backward. The universe isn't a reel of film, there is no cosmic computer storing an array of state vectors indexed in time. Even if the film was run in reverse, each sequence would need to be calculated afresh and the system will then evolve ergodically, forwards or backwards. It is a mistake to think the state vector would be the same either way - the result is incomputable and unknowable. Thus there is no disparity between microscopic laws and macroscopic irreversibility.

To conclude, on the author's current state of experimental enquiries (let alone other reports by other parties[5,9-12]), unless there is some deux ex-machina, along the lines of suspending kinetic theory (so that the ferrofluid working substance won't relax magnetically) or some conspiracy with electromagnetic theory (to avoid the transfer of power), given the favourable analysis of losses in the system, a completed device would most likely show the effect predicted.

This work has been supported, primarily by a private research grant from a private investor (Richard Edward Balding), the author's input and the South Western Regional Development Association (SWRDA) and supervised at the Future Energy Group, The University of London, Queen Mary.

\section{Appendix A. 1st order phase change demon}

Originally a column with an hygroscopic solution was envisioned (fig 9a,b) where the head of water condensed at height would be sufficient to force water out of a reverse osmosis membrane. This setup was meant to make a "phase changing catalyst" (fig 9a) which would reside in a closed, isolated system (with gravity) containing water in the liquid and vapour phases (fig. 10).

There are two processes occurring: A continuous conversion of heat to work in the field from the external potential of the vapour rising in the gravitational field. As considered in the main text, microscopic heat is constantly converted into microscopic work to allow the gas to rise in gravity field at constant temperature. This work ultimately provides the head on the column to separate the 
water from the hygroscopic liquid. The other $1^{\text {st }}$ order change collects heat from the lower reservoir by the discontinuous phase change at constant temperature and then is able to liberate the heat as a temperature difference, by partial condensation of the vapour.

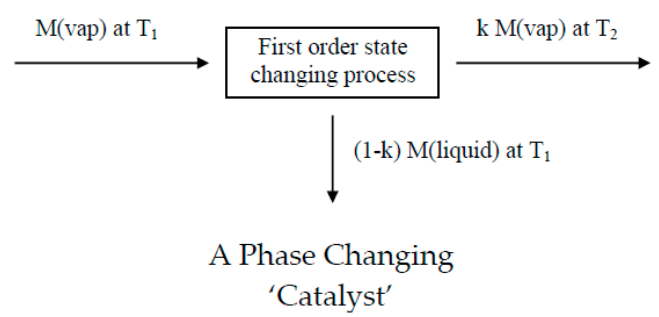

(a)

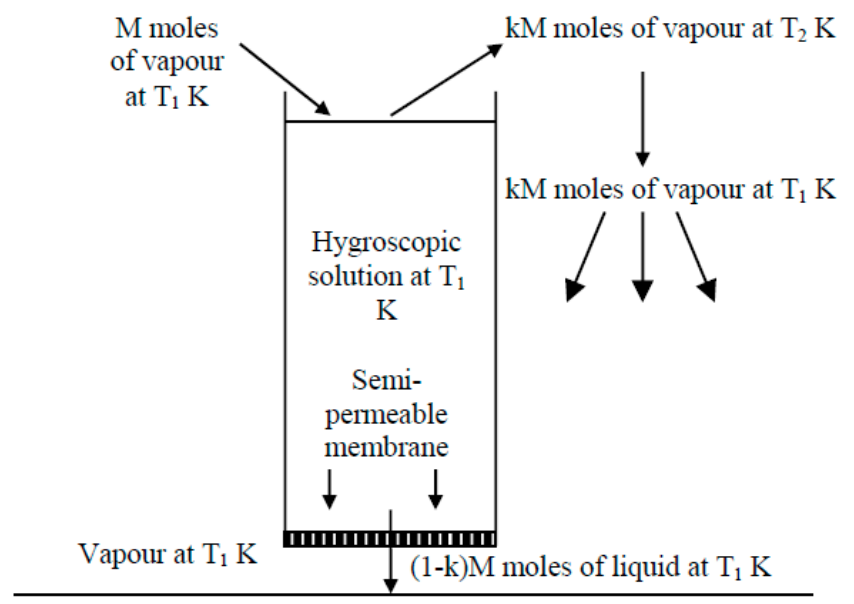

Liquid at $\mathrm{T}_{1} \mathrm{~K}$

(b)

Figure 9a,b. Hygroscopic "phase changing catalyst" column demon

The analysis follows (w.r.t. fig 9a); it is learnt that the column would be very high (though there is still enough vapour pressure to drive water into the solution at the top of the column). Flow losses would realistically result in a trickle of water at the base and render the device impractical for power generation but a paradox would still remain (fig. 10).

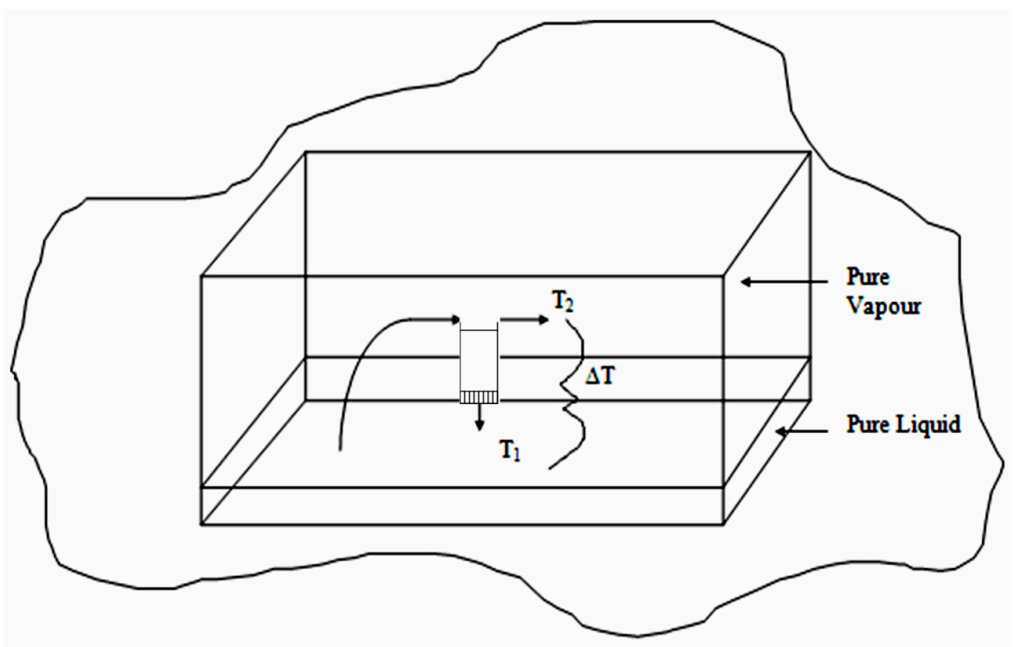

Closed, Isolated Universe

Figure 10. Device in two phase system 
Let the process occur at constant pressure. For the First Law modelling, I can use enthalpy:-

$$
H=U+P V
$$

By the conservation of energy (1st Law), w.r.t fig 9a:

Enthalpy of reactants before process = Enthalpy of reactants after.

Enthalpy of $\mathrm{M}$ (vapour) at $\mathrm{T}_{1}$ Kelvin

$$
\Delta H_{1}=M C_{L}\left(T_{1}-T_{x}\right)+M L
$$

where

$C_{L}$ is the specific heat capacity of the liquid phase

$\mathrm{T}_{\mathrm{x}}$ is a dummy temperature variable

$\mathrm{L}$ is the latent heat of phase transition

Enthalpy of (1-k) M(liquid) at $\mathrm{T}_{1}$ Kelvin, where $\mathrm{k}$ is a mass fraction

$$
\Delta H_{2}=(1-k) M C_{L}\left(T_{1}-T_{x}\right)
$$

Enthalpy of $\mathrm{k}$ M(vapour) at $\mathrm{T}_{2}$ Kelvin

$$
\Delta H_{3}=k M C_{L}\left[\left(T_{1}-T_{x}\right)+L\right]+k M C_{P}\left(T_{2}-T_{1}\right)
$$

where $C_{P}$ is the specific heat capacity of the vapour phase

By 1st Law $\Delta \mathrm{H}_{1}=\Delta \mathrm{H}_{2}+\Delta \mathrm{H}_{3}$ and eliminating $\mathrm{T}_{\mathrm{x}} \Rightarrow T_{2}-T_{1}=\frac{(1-k)}{k} \frac{L}{C_{P}}$

A.1. A temperature rise is predicted by the First Law. The second law shall be used to calculate $k$.

For the Second Law modelling of the "phase changing catalyst" the process is broken into two steps:

Step 1: Heat withdrawn as part of gas is condensed, (1-k) M(vapour) $\rightarrow$ (1-k) M(liquid)

Change in entropy at constant temperature:

$$
\Delta S_{1}=-(1-k) \frac{M L}{T_{1}}
$$

Step 2: Heat added as remaining vapour heated, $\mathrm{kM}$ (vapour) at $\mathrm{T}_{1} \rightarrow \mathrm{k} \mathrm{M}$ (liquid) at $\mathrm{T}_{2}$ Change in entropy of substance behaving like ideal gas to an approximation:

$$
\Delta S_{2}=k M C_{P} \ln \left(\frac{T_{2}}{T_{1}}\right)
$$

The total change in entropy is $\Delta S_{1}+\Delta S_{2} \geq 0$ by 2nd Law. Working the inequality,

$$
k \leq \frac{L}{L+T_{1} C_{P} \ln \left(\frac{T_{2}}{T_{1}}\right)}
$$

A.2. A temperature rise is permitted by the Second Law as (1-k), the mass fraction of water condensed can be greater than zero (eqn. 23), the condensation of the water releases latent heat (eqn. 20).

Practically we concern ourselves with the fall of saturated vapour pressure with height and then see if a tall column of hygroscopic solution would: a) have enough head to act against the osmotic pressure of the solution to release water at the bottom, b) wouldn't be too tall, so that water vapour wouldn't condense at the top.

Equating the head of the column to the osmotic pressure:

$$
\begin{gathered}
h \rho g \geq c R T \\
h \geq \frac{c R T}{\rho g}
\end{gathered}
$$

where: $\mathrm{h}$ is the height of the column, $\mathrm{g}$ is the gravitational acceleration, $\rho$ is the density of the hygroscopic solution, $\mathrm{R}$ is the molar gas constant and $\mathrm{T}$ is the absolute temperature. The Osmotic pressure formula is only accurate for dilute solution but we are attempting a "ball-park" calculation.

The Barometric pressure law is:

$$
P_{h}=P_{0} e^{-\frac{M g h}{R T}}
$$


where: $\mathrm{P}_{h}$ is the water vapour pressure at height $h, \mathrm{P}_{0}$ is the vapour pressure at the bottom of our apparatus, $\mathrm{M}$ is the molar mass of the water vapour.

TABLE B.1 Saturated Water: Temperature Table

\begin{tabular}{llccrr}
\hline $\begin{array}{l}T \\
{ }^{\circ} \mathrm{C}\end{array}$ & $\begin{array}{c}P \\
\mathrm{kPa}, \mathrm{MPa}\end{array}$ & $\begin{array}{c}\hat{v}_{l} \\
\mathrm{~m}^{3} / \mathrm{kg}\end{array}$ & $\begin{array}{c}\hat{v}_{\mathrm{v}} \\
\mathrm{m}^{3} / \mathrm{kg}\end{array}$ & $\begin{array}{r}\hat{u}_{l} \\
\mathrm{kj} / \mathrm{kg}\end{array}$ \\
\hline 0.01 & 0.6113 & 0.001000 & 206.132 & 0.00 & 4 \\
5 & 0.8721 & 0.001000 & 147.118 & 20.97 & 4 \\
10 & 1.2276 & 0.001000 & 106.377 & 41.99 & 4 \\
15 & 1.7051 & 0.001001 & 77.925 & 62.98 & 4 \\
20 & 2.3385 & 0.001002 & 57.790 & 83.94 & 4 \\
25 & 3.1691 & 0.001003 & 43.359 & 104.86 & 4 \\
30 & 4.2461 & 0.001004 & 32.893 & 125.77 & 4 \\
35 & 5.6280 & 0.001006 & 25.216 & 146.65 & 4 \\
40 & 7.3837 & 0.001008 & 19.523 & 167.53 & 4 \\
45 & 9.5934 & 0.001010 & 15.258 & 188.41 & 4
\end{tabular}

Figure 11. Saturated Steam Tables: "Engineering and Chemical Thermodynamics", John Wiley \& Sons, 2004.

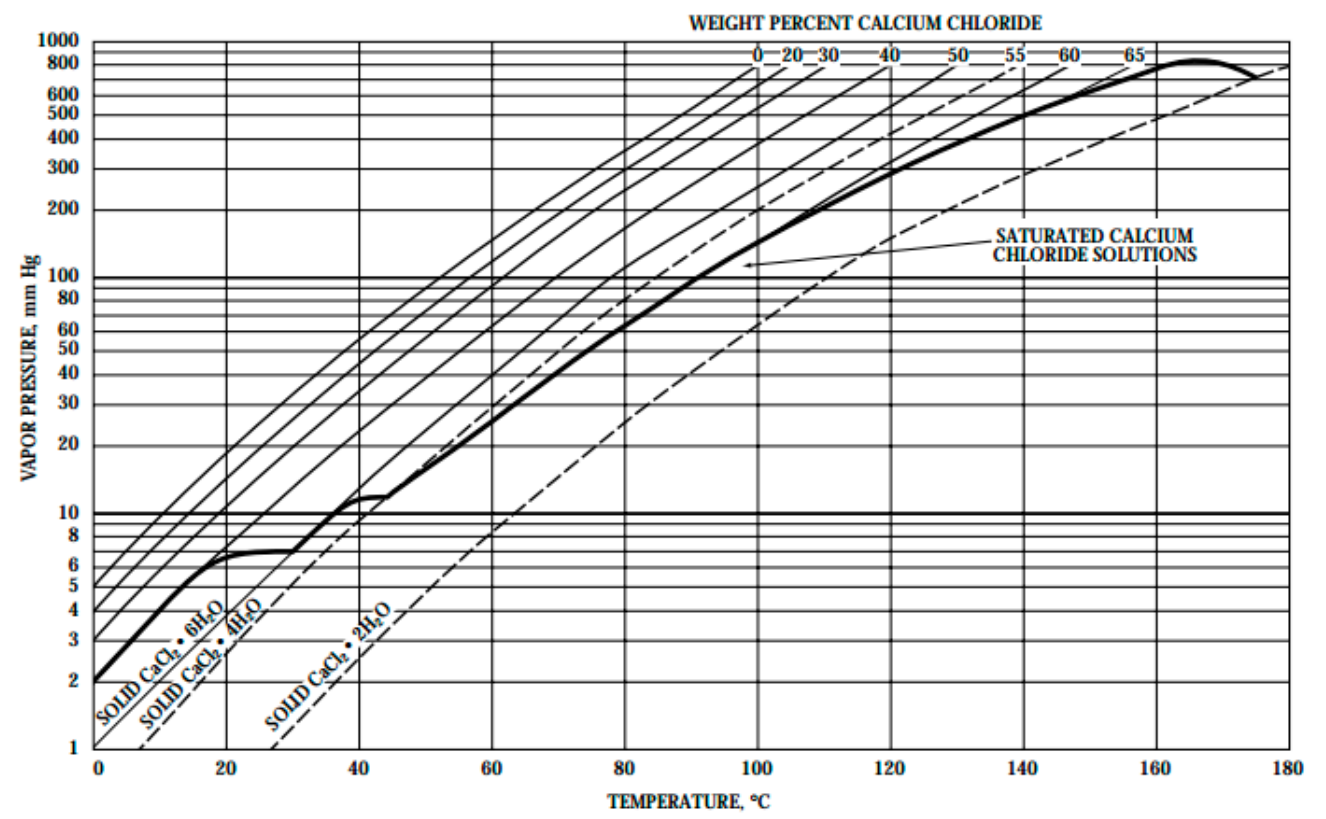

Figure 12. Calcium Chloride Handbook, Dow Chemicals 2003.

If we substitute eqn. 24 into eqn. 25 , the minimum pressure at height must be:

$$
P_{h} \geq P_{0} e^{-M \frac{C}{\rho}}
$$

Considering the water vapour pressure at $20^{\circ} \mathrm{C}$ of about $2.34 \mathrm{KPa}$, the molar mass of water is $0.018 \mathrm{Kg}$ and then the $30 \% \mathrm{w} / \mathrm{w} \mathrm{CaCl}_{2}(\mathrm{aq})$, which is a solution by the chart above, would make the molar concentration of $\mathrm{CaCl}_{2}$ some 2703 moles $/ \mathrm{m}^{3}$ and the density of the solution would be $1300 \mathrm{Kg} / \mathrm{m}^{3}$. We find the minimum vapour pressure at height for the column to function is: $2.26 \mathrm{KPa}$. The vapour pressure of water above for $30 \% \mathrm{w} / \mathrm{w} \mathrm{CaCl}_{2}(\mathrm{aq})$ at $20^{\circ} \mathrm{C}$ is about $1.33 \mathrm{KPa}$ but it is enough to look at the $0 \% \mathrm{w} / \mathrm{w} \mathrm{CaCl} 2$ line (i.e. pure water) and see that it is greater. Therefore the process occurs. 
The osmotic pressure of the $\mathrm{CaCl}_{2}$ solution at $30 \% \mathrm{w} / \mathrm{w}$, some $2.7 \mathrm{M}$ or three times this with the number of ions, $8.1 \mathrm{M}$ has an osmotic pressure of nearly $200 \mathrm{atms}$ and this is the lower bound by the simple Van t'Hoff law. It would be difficult to contemplate building a column high enough to provide sufficient head for the separation. Another approach is to place the hygroscopic liquid in a semipermeable vesicle, which would swell, such that the internal pressure would then provide the head. These thoughts come full circle with the author's initial intent, several years ago, to design improved water desalination techniques. It was the enquiry into the figure of merit for the process that gave the clue to unusual thermodynamics.

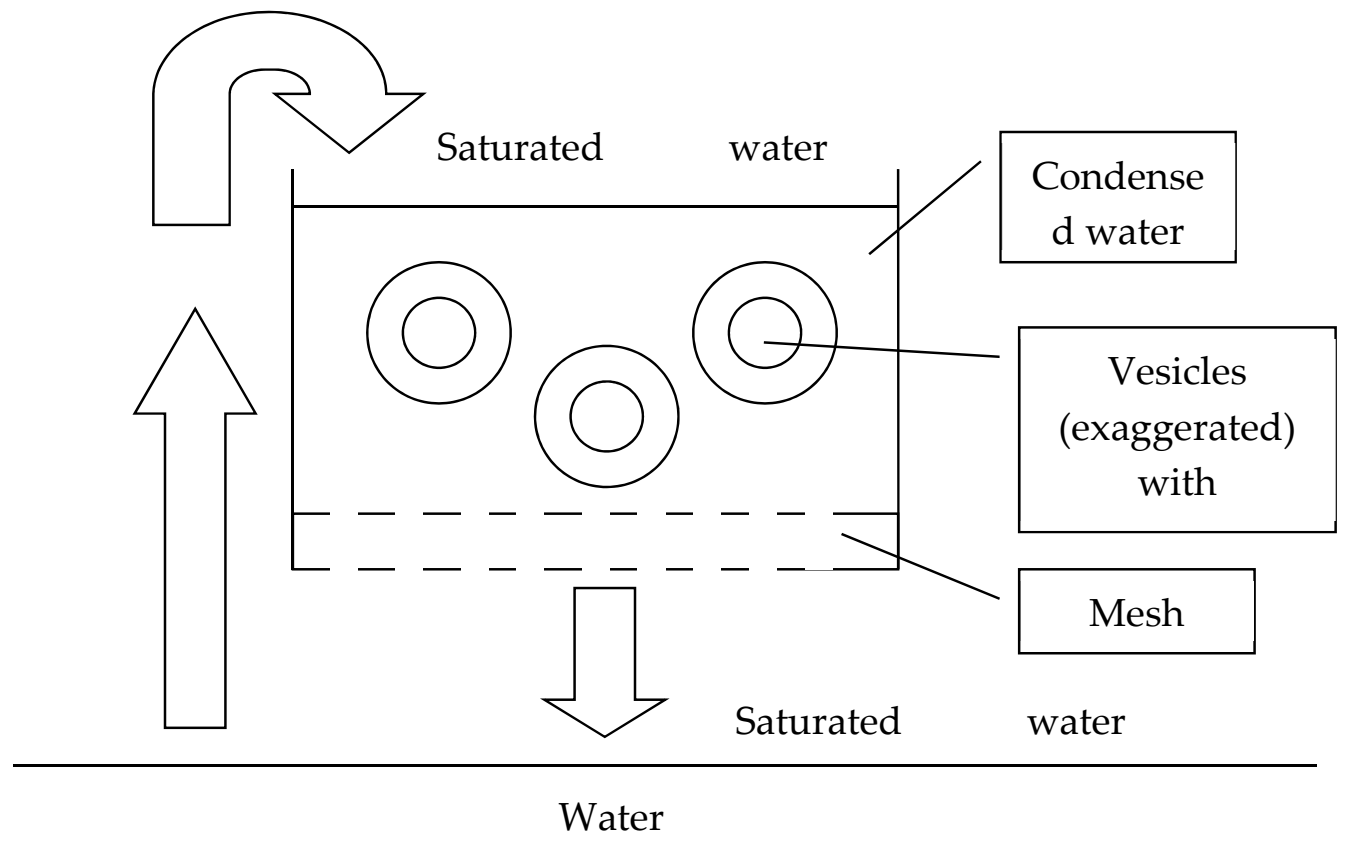

Figure 13. The author's initial water desalination scheme

\section{References}

1. Maxwell, J.C., Theory of Heat. Longmans, Green and Co., London Vol. Chap. 12. 1871.

2. Szilard, L., On the Decrease of Entropy in a Thermodynamic System by the Intervention of Intelligent Beings. Physik Z., 1929. 53: p. 840-856

3. Jaynes, E.T., The muscle as an engine. 1983 (http://bayes.wustl.edu/etj/articles/muscle.pdf).

4. Ho, Mae.-Wang., The Rainbow and the Worm: The Physics of Organisms. 2nd ed: World Scientific.

5. V. Čápek, D.P.Sheehan., Challenges to the Second Law of Thermodynamics. 2005: Springer.

6. Landau, L., A Course in Theoretical Physics: Statistical Physics. Vol. Vol. 5. 1996: Butterworth Heinemann.

7. Landau, L., A Course in Theoretical Physics: Kinetics. Vol. Vol. 10. 1981: Butterworth-Heinemann.

8. Kittel C., Kroemer.H., Thermal Physics. W. H. Freeman and Company, San Francisco. Vol. 2nd ed. 1980.

9. D.P. Sheehan, D.J.Mallin.et.al., Experimental Test of a Thermodynamic Paradox. Found Phys, 2014. 44.

10. D'Abramo, G., Thermo-charged capacitors and the Second Law of Thermodynamics. Phys. Lett. A, 2010. 374: p. 1801-1805.

11. Sheehan, D.P., The Second Law of Thermodynamics:

Foundations and Status. Found Phys, 2007. 37: p. 1653-1658.

12. Santhanam, P., D.J. Gray, and R.J. Ram, Thermoelectrically Pumped Light-Emitting Diodes Operating above Unity Efficiency. Physical Review Letters, 2012. 108(9): p. 097403.

13. Abbott M. M., Van-Ness H.G., Thermodynamics with Chemical Applications. Schaum's Outlines, McGraw-Hill. 1989.

14. Smoluchowski, M., Experimentell nachweisbare, der Ublichen Thermodynamik widersprechende Molekularphenomene. Phys. Zeitshur., 1912. 13.

15. Brillouin, L., Science and Information Theory. Academic Press Inc., New York 1956. Chaps. 13, 14. 
16. Feynman, Leighton., Sands, The Feynman Lectures on Physics. Addison-Wesley, Reading, Massachusetts. Vol. Vol.1, Vol. 2 1989. 14.7, 15.1-6, 35.4

17. Harvey L. S., Andrew.F.R., Maxwell's Demon: Entropy, Information and Computing. Adam Hilger, Bristol 1991.

18. Cornwall R. O., Novel Thermodynamic Cycles involving Ferrofluids displaying Temporary Magnetic Remanence. 2013 (http://vixra.org/abs/1311.0078).

19. Rosensweig, R.E., Ferrohydrodynamics. Cambridge University Press. 1998.

20. Haile J. M., Molecular Dynamics Simulation. 1997: John Wiley and Sons.

21. Cornwall, R.O., How to build a Maxwell Demon from a 2nd order Phase Change System. 2013 (http://vixra.org/abs/1311.0077).

22. Kapitaniak, T., Chaos for Engineers. Springer 1998.

23. Jordan D. W., Smith.P., Nonlinear Ordinary Differential Equations. Clarendon Press Oxford. Vol. 2nd ed. . 1991.

(C) 2017 by the authors. Licensee Preprints, Basel, Switzerland. This article is an open access article distributed under the terms and conditions of the Creative Commons by Attribution (CC-BY) license (http://creativecommons.org/licenses/by/4.0/). 